\title{
Observaciones adicionales sobre estadística en el programa Epiinfo 6.0
}

\author{
Gastón Duffau T.'
}

\begin{abstract}
Resumen
Se presentan observaciones sobre los subprogramas esladisticos Stalcalc y Epirable del programa Epinto versión s.o. 1994. En lo orincipal. se advierten pesiteles problenas para el usuario al emplear las rutinas de comparación de popcraiones, ya cue aun con menos de 20 cosos efectúc a pruebo de chi cuadrado; cálculo de ventaja |OR| para los que no siempre corcuerdan los resullados de Stetcác y Epitable; inlenvalo de confianza de la med cno, que genera sólo posiciones en una curva normel y de prosorciones $\mathrm{C} \%$ y $100 \%$; tamaño muestrol para un estudio easocontrol, en que las sub'utinas estadísticas proporcionsn 'esullados muy difeientes; se ección de numeros al azar, que no permite crdenor un corijunlo dado de números: cálculo del poder en estudic de cchortes, que puede señalcr error bela de cero y cnálisis de una pruebo diagłóstica que no mencio za estóndar ideal, prevalencia ni validez.
\end{abstract}

[Palabras clave: estadistica, biometrío, programos comoutadorizados.)

\section{Epiinfo 6.0 statistical software. Further observations}

Further revew of statistica! programme in Esinfo 00 is presented. Several problems were abserved, manly when operating Stacale and Epitable rov'ines in the followirg cases; winen making comparissons between wo proportions chil squared s perfomed gven with less than 20 cases; adds raios $195 \%$ confidence intervalt results are different in Siacolc cnd spicuble; when calculctions for confidence interval of redian wolues cre done, only possilions in an abnormol curve and proportions of $0 \%$ and $100 \%$ are given. estimations for somple sizes in casecontro studies are quite differen in Statcale ond Epitable; random number selection is offered where randomization of o given set $0^{*}$ - umbers is not feasible; power colculations are dona in cohort siudies where $0 \%$ bera error is possible; 'ests for sc'eening without eny mention to gold stondard, pevalence or validity are offered.

'Key words: slatistics, bioneiry. epidemiological methods, sofkwcre.|

Epiinfo es un "programa procesador de palabras, base de datos y estadístico para salud pública". Se dispone de csle instrumento de trabajo sin costo alguno para el interesado y es de notable utilidad por cuanto contiene las rutinas de mayor uso por el investigador clínico. Algunas de ellas fueron previamente sometidas a un análisis críticol.

El objetivo de este estudio es describir nuevas observaciones, consideradas de interés para

1. Departamento de Pediatría y Cirugía Infantil, Campus Norte, Facuitad de Medicina. Universidad de Chile.

Con financiamiento de Proyecto Fondecyt $\mathrm{N}^{\circ} 19,50656$. el usuario, sobre los subprograrnas estadísticos Statcalc y Epitable, tomando en consideración que si bien el que va a utilizar este programa debiera estar familiarizado con él, no es menos cierto que resulta improbable que se pueda contar con que el investigador efectúe una revisión del mismo antes de emplearlo.

\section{Material y Método}

Se dispuso de Epiinfo 6.0 (1994) y 6.04 (1996) y se revisaron otras secciones de los subprogramas estadísticos Statcalc y Epitable, al que se presentaron diversos problemas a re- 
solver, escogiendo siempre las rutinas probablemente más empleadas por investigadores clínicos, con la intención de hacer al lector algunas advertencias que pudieran serle útiles al proce. sar con este instrumento los datos de una investigación. Los nombres de las opciones que ofrece el programa se han dejado casi siempre en el idioma original, inglés, para facilitar su localización y evitar confundir al usuario. Cuando corresponde, se indican las correcciones que pudiera haber introducido la versión más reciente de aquél.

\section{Resultados y Comentario}

Epitable, en la comparación de dos proporciones - donde utiliza Ji cuadrado-, construye una tabla (" $\mathrm{x}$ x c ") con cifras que pueden sumar un total inferior a $20 \mathrm{y}$ no recomienda efectuar en su reemplazo la prueba de Fisher de probabilidades exactas ${ }^{2,3}$. Es más, acepta que en la totalidad de las celdas existan valores esperados inferiores a 5 y la única reacción observada es que aplica corrección de Yates ${ }^{3,4}$. Tal es la situación frente a una tabla de $2 \times 2$ como la siguiente:

$$
\begin{array}{ll}
0 & 5 \\
4 & 0
\end{array}
$$

En ésta, indica Ji cuadrado con corrección de Yates $=5,41$ y $p=0,02$, debiendo obtener por Fisher $p=0,007$.

En la rutina "Study-case.control", Epitable proporciona el cálculo de ventaja ("odds ratio" (OR)). ( $N$. del a.: esta expresión ha sido traducida de muy diversas maneras y no parece existir acuerdo acerca de aquélla más apropiada: "ventaja", "tazón de desigualdades", "razón de productos cruzados, "desigualdad relativa", "razón de la diferencia", "razón de la probabilidad", "razón de ventaja", "razón de disparidad", "razón de nomios", "posibilidades relativas", "razón de posibilidades" y otras ${ }^{5.7}$ ) pero no produce el mismo resultado que mediante Statcalc. En efecto, en la tabla:

$$
\begin{array}{ll}
71 & 52 \\
29 & 48
\end{array}
$$

Statcalc último indica que $O R$ es 2,26 con intervalo de confianza (IC) de $95 \%$ (IC 95\%) de 1,21 - 4,23, mientras Epitable, para una situación si- milar de casos no pareados, señala OR 2,26 con IC 95\% de 1,26 - 4,05 (1,25 - 4,06 en la versión 6.04). S6lo al obtener el intervalo "exacto" de confianza coinciden los dos resultados.

Epitable, para el cálculo del intervalo de confianza (95\%) de una proporción, método cuadrático de Fleiss ${ }^{2}$, propone una formula en la opción "Ayuda", que al utilizarla no permite establecer que el límite superior es $100 \%$ y el inferior $0 \%$ en la eventualidad que la muestra analizada indique, respectivamente, proporciones de $100 \%$ o $0 \%$. Sin embargo, el programa en tales casos muestra en pantalla correctamente los límites señalados, cuando corresponde. El programa, entonces, no emplea en esas oportunidades la solución sugerida en la opción "Ayuda". Esto se podría ver apoyado, además, por el hecho que cuando la proporción es $0 \%$ o $100 \%$ la respuesta no demora especialmente, pero cuando es cercana a esas cifras el programa tarda mucho más. La situación es parecida a lo que ocurre con el estudio de eficacia de vacunas (cohortes), en donde si bien la pantalla proporciona un resultado correcto, la fórmula propuesta en la opción "Ayuda" para el cálculo del riesgo relativo es errónea'.

En el cálculo del intervalo de confianza $(95 \%)$ de la mediana ${ }^{8}$ en una serie, se establece como requisitos que la variable tenga distribución normal y el efectivo de la muestra sea mayor que 60 observaciones. Si se dan las condiciones mencionadas no parece en absoluto necesario trabajar con la mediana, que por lo demás debiera coincidir con el promedio. Aunque hay una variedad de operaciones estadísticas que pueden realizarse con la mediana, no son técnicas particularmente empleadas por investigaciones en el área médica. En inferencia estadística parece improbable que pueda desplazar al promedio para distribuciones en que éste se encuentre indicado. Por otra parte, no hay que equivocarse pues el programa entrega la posición mediana y no el valor de ésta. Lo mismo ocurre con las cifras del IC $95 \%$.

En la rutina "sample-sample size-single proportion", en que es posible tener una estimación del tamaño muestral apropiado para estudiar una prevalencia en una población delerminada, se requiere conocer: el tamaño de la población de la cual se extraerá la muestra, lo que puede ser un serio problema; la precisión deseada; la prevalencia esperada en esa pobla- 
ción (si no se tiene una cierla idea no se puede efecruar el cálculo y si aquélla es muy equivocada, el tamaño de la muestra propuesto tambièn lo será); el nivel de confianza de la estimación; el efecto del diseño (éste se conoce aproximadamente para la situación que se estudia). Al respecto es importante recordar que todo cálculo de tamaño muestral es sólo una aproximación razonable, ya que debe estar funJada en un conjunto de presunciones, hecho que aquí parece quedar bastante claro. En la opción "Ayuda" aparece la fórmula utilizada en el cálculo, pero la forma en que se deben emplear los datos numéricos difiere de la solicitada por la pantalla del programa. Así, para datos hipotéticos éstas serían respectivamente:

\section{Fórmula Pantalla (\%)}

$\begin{array}{llr}\text { Precision deseada } & 0,033 & 3 \\ \text { Prcvalencia esperada } & 0,1515 & 15 \\ \text { Nivel de confianza } & 1,965 & 5\end{array}$

En el nivel de confianza la fórmula emplea el valor " $z$ " $(1,96)$ que corresponde a $95 \%$ de confianza. pero la pantalla pide el riesgo de $5 \%$.

Para calcular el tamaño de la muestra en un diseño caso-control no pareado. se puede recurrir a Statcalc o Epitable, pero se obtendrán resultados distintos. Así por ejemplo, con los siguientes datos:

Relación controles/casos: 1; OR más pequeño que interese detectar: 2,$5 ; \%$ de exposición entre los controles: 5,0 ; error alfa: $5 \%$; poder: 80\%, Statcalc requeríá 301 casos e igual nómero de controles y Epitable 118 casos y 118 controles. Este error está corregido en la versión 6.04 de Epiinfo. donde se obtiene como resultado 301 casos y 301 controles en ambos programas.

En la rutina "sample-power calculationcohort study", sorprendentemente se puede llegar a un error beta de cero y por tanto a un poder de $100 \%$. Así, una investigación que incluyera dos muestras de 750 casos con relación expuestos/no expuestos de $1: 1$. un riesgo relativo de interćs para el investigador de $2,740 \mathrm{ma}$ yor, una tasa de ataque en aquellos no expuestos de $4,3 \%$ y un enor alfa de $5 \%$, tendría un poder de cien por ciento, es decir sería nula la probabilidad de no detectar un riesgo relativo de 2,74 o mayor.
Los números al azar en "sample-random number" son ofrecidos en dos formas: como tabla o como una lista de números al azar. En esta última se puede solicitar un conjunto de números a ser elegidos de un grupo mayor, pero no se puede obtener el ordenamiento al azar, por ejemplo, del 1 al 50, sin reposición. Si se hace, dando como posibilidad la elección de cifras del I al 55, aparece un número muy elevado de grupos en unas pocas hileras:

[-2-3-4-5-6-7-8-9-10-1]

13-14-15-16-17-18-19-20

$22-23-24-25-26-27-28-29-30-31-32-33-34-35-36-37$

$39-40-41$

$43-44-45-46-47-48-49-50-51-52$

54-55

Aplicando runs test para una muestra ${ }^{4}$, el hallazgo resulta muy improbablemente explicable por el azar.

Por otra parte, en "study-screening" se analizan las propiedades de una prueba diagnóstica en términos de sensibilidad, especificidad y valores predictivos, todos con sus límites de conflanza según Fleiss ${ }^{2}$, pero no se mencionan en forma explícita elementos tan importantes como: estándar ideal o patrón de oro (gold standard), prevalencia del problema en estudio y validez de la prueba.

En lo principal, el investigador clínico que va a utilizar el conjunto Epiinfo 6 deberá estar informado de las peculiaridades de cada rutina estadística, pues involuntaria e inadvertidamente puede errar o interpretar mal varias de ellas: comparación de proporciones, indicación de la corrección de Yates, cálculo del intervalo de confianza de "odds ratio", de una proporción y de la mediana determinación del tamaño muestras para el estudio de una prevalencia o en un diseño caso-control, cálculo del poder en un estudio de cohorte, oblención de números al azar y características de una prueba diagnostica.

\section{Referencias}

1. Duffor $G$ : Estadística en el programa Epiinfo 6.0. Rev Chil Pediatr 1995; 66: 341-344.

2. Flcirr J: Statistical methods for rates and proportions. and edition, New York: John Wiley \& Sons 1980: 56 . 82 . 
3. Marthews $D$. Farewell V: Estadística médica. Aplicación interpretación. Barcelona: Salvat Editores S.A. 1988: 11-66.

4. Siegel S: Nonparametric staristics for the behavioral sciences. New York: McGraw-Hill Book Co. 1956: 52. 60.

5. Bautisitt LE: "Razón relativa" y "tasa relativa" como traducciones de odds matio $y$ hazard ratio. Bol Oficina Sanit Pariam 1995: 119: 278-279.
6. Ingelfinger J. Mosteller $F$, Thibodeau L, Ware J: Biostatistics in clínica] medicine, 2nd edition, New York: Macmillan Publishing Co., 1987: 168-180

7. Valenzuela $C$ : Dos soluciones para la estimación de odds ratios con ceros. Rev Med Chile 1993; 121 : 1441 1444.

B. Sieel R. Torrie J: Bioestadística: Principios y procedimientos. Madrid: McGraw-Hill 1990: 102-105. 